\title{
Antifibrotic effect of xanthohumol in combination with praziquantel is associated with altered redox status and reduced iron accumulation during liver fluke-associated cholangiocarcinogenesis
}

Wassana Jamnongkan ${ }^{1,2}$ ， Malinee Thanee ${ }^{1,2}$ ， Puangrat Yongvanit ${ }^{1,2}$, Watcharin Loilome ${ }^{1,2}$, Raynoo Thanan 2, Phongsaran Kimawaha ${ }^{2,3}$, Tidarat Boonmars ${ }^{2,4}$, Runglawan Silakit ${ }^{1,2}$, Nisana Namwat ${ }^{1,2}$, Anchalee Techasen ${ }^{\text {Corresp. } 2,3}$

${ }^{1}$ Department of Biochemistry, Faculty of Medicine, Khon Kaen University, Khon Kaen, Thailand

2 Cholangiocarcinoma Research Institute, Khon Kaen University, Khon Kaen, Thailand

3 Faculty of Associated Medical Sciences, Khon Kaen University, Khon Kaen, Thailand

4 Department of Parasitology, Faculty of Medicine, Khon Kaen University, Khon Kaen, Thailand

Corresponding Author: Anchalee Techasen

Email address: anchte@kku.ac.th

Cholangiocarcinoma (CCA) caused by infection by the liver fluke Opisthorchis viverrini, (Ov) is the major public health problem in northeast Thailand. Following Ov infection the subsequent molecular changes can be associated by reactive oxygen species (ROS) induced chronic inflammation, advanced periductal fibrosis, and cholangiocarcinogenesis. Notably, resistance to an activation of cell death in prolonged oxidative stress conditions can occur but some damaged/mutated cells could survive and enable clonal expansion. Our study used a natural product, xanthohumol (XN) which is an anti-oxidant and antiinflammatory compound to examine whether it could prevent Ov-associated CCA carcinogenesis. We measured the effect of XN with or without praziquantel (PZ), an antihelminthic treatment, on DNA damage, redox status change including iron accumulation and periductal fibrosis during CCA genesis induced by administration of Ov and $\mathrm{N}$ dinitrosomethylamine (NDMA) in hamsters. Animals were randomly divided into four groups: group I, Ov infection and NDMA administration (ON); group II, Ov infection and NDMA administration and PZ treatment (ONP); the latter 2 groups were similar to group I and II, but group III received additional XN (XON) and group IV received XN plus PZ (XONP). The results showed that high 8-oxodG (a marker of DNA damage) was observed throughout cholangiocarcinogenesis. Moreover, increased expression of CD44v8-10 (a cell surface in regulation of the ROS defense system), whereas decreased expression of phospho-p38 ${ }^{\text {MAPK }}$ (a major ROS target), was found during the progression of the bile duct cell transformation. In addition, high accumulation of iron and expression of transferrin receptor-1 (TfR-1) in both malignant bile ducts and inflammatory cells were detected. 
Furthermore, fibrosis also increased with the highest level being on day 180 . On the other hand, the groups of XN with or without PZ supplementations showed an effective reduction in all the markers examined, including fibrosis when compared with ON group. In particular, the XONP group in which a significant reduction DNA damage occured, was also found to have iron accumulation and fibrosis compared to the other groups. Our results show that XN administered in combination with PZ could efficiently prevent CCA development and hence provide potential chemopreventive benefits in Ov-induced cholangiocarcinogenesis. 
1 Antifibrotic effect of xanthohumol in combination with praziquantel is associated with

2 altered redox status and reduced iron accumulation during liver fluke-associated

3 cholangiocarcinogenesis

4 Wassana Jamnongkan ${ }^{1,4}$, Malinee Thanee ${ }^{1,4}$, Puangrat Yongvanit ${ }^{1,4}$, Watcharin Loilome ${ }^{1,4}$,

5 Raynoo Thanan ${ }^{1,4}$, Phongsaran Kimawaha ${ }^{2,4}$, Tidarat Boonmars ${ }^{3,4}$, Runglawan Silakit ${ }^{1,4}$, Nisana

6 Namwat $^{1,4}$ and Anchalee Techasen ${ }^{2,4 *}$

$7 \quad{ }^{1}$ Departments of Biochemistry, ${ }^{3}$ Parasitology, Faculty of Medicine, Khon Kaen University, Khon

8 Kaen, Thailand.

$9{ }^{2}$ Faculty of Associated Medical Sciences, Khon Kaen University, Khon Kaen, Thailand.

$10{ }^{4}$ Cholangiocarcinoma Research Institute, Khon Kaen University, Khon Kaen, Thailand.

\section{Address correspondence to:}

12 Dr. Anchalee Techasen

13 Faculty of Associated Medical Sciences, Khon Kaen University, Khon Kaen, Thailand. Phone

$14+6643202086$, Telefax +6643202086

15 E-mail address: anchte@kku.ac.th 


\section{ABSTRACT}

Cholangiocarcinoma (CCA) caused by infection by the liver fluke Opisthorchis viverrini,

$(\mathrm{Ov})$ is the major public health problem in northeast Thailand. Following Ov infection the

subsequent molecular changes can be associated by reactive oxygen species (ROS) induced chronic inflammation, advanced periductal fibrosis, and cholangiocarcinogenesis. Notably,

resistance to an activation of cell death in prolonged oxidative stress conditions can occur but some damaged/mutated cells could survive and enable clonal expansion. Our study used a natural product, xanthohumol $(\mathrm{XN})$ which is an anti-oxidant and anti-inflammatory compound to examine whether it could prevent Ov-associated CCA carcinogenesis. We measured the effect of $\mathrm{XN}$ with or without praziquantel (PZ), an anti-helminthic treatment, on DNA damage, redox status change including iron accumulation and periductal fibrosis during CCA genesis induced by administration of $\mathrm{Ov}$ and $\mathrm{N}$-dinitrosomethylamine (NDMA) in hamsters. Animals were randomly divided into four groups: group I, Ov infection and NDMA administration (ON); group II, Ov infection and NDMA administration and PZ treatment (ONP); the latter 2 groups were similar to group I and II, but group III received additional XN (XON) and group IV received XN plus PZ (XONP). The results showed that high 8-oxodG (a marker of DNA damage) was observed throughout cholangiocarcinogenesis. Moreover, increased expression of CD44v8-10 (a cell surface in regulation of the ROS defense system), whereas decreased expression of phospho-

$34 \mathrm{p} 38^{\mathrm{MAPK}}$ (a major ROS target), was found during the progression of the bile duct cell transformation. In addition, high accumulation of iron and expression of transferrin receptor-1 (TfR-1) in both malignant bile ducts and inflammatory cells were detected. Furthermore, fibrosis also increased with the highest level being on day 180. On the other hand, the groups of XN with or without PZ supplementations showed an effective reduction in all the markers examined, 
40 significant reduction DNA damage occured, was also found to have iron accumulation and

41 fibrosis compared to the other groups. Our results show that $\mathrm{XN}$ administered in combination

42 with PZ could efficiently prevent CCA development and hence provide potential

43 chemopreventive benefits in Ov-induced cholangiocarcinogenesis.

\section{INTRODUCTION}

45 Chronic inflammation induced by liver fluke (Opisthorchis viverrini, Ov) infection is the 46 major risk factor for cholangiocarcinoma (CCA) in northeast Thailand. Re-infection of people in

47 endemic areas occurs more likely after praziquantel treatment and frequently leads to periductal

48 fibrosis that governs the pathogenic effects of inflammation and immunity in liver fluke-

49 associated cholangiocarcinogenesis has been recently reviewed (Yongvanit et al. 2012a). Cellular

50 damage caused by reactive oxygen species (ROS) and reactive nitrogen species (RNS) during

51 chronic infection appears to be a key event which is further enhanced by an imbalanced

52 oxidant/anti-oxidant system during reinfections (Yongvanit et al. 2012b). Increase in periductal

53 fibrosis has been shown to be associated with CCA genesis (Prakobwong et al. 2009). After Ov

54 infection, time-dependent periductal fibrosis has been detected with subsequent CCA

55 development (Chamadol et al. 2014; Mairiang et al. 1992).

56 Recently, studies have shown that the expression of CD44 variants form (CD44v) could

57 stabilize $\mathrm{xCT}$ (cysteine-glutamate transporter) which promotes cystine uptake and then its

58 conversion to cysteine contributing to glutathione synthesis for ROS defense (Ishimoto et al.

59 2011; Kim et al. 2002). Moreover, Thanee et al. showed that an accumulation of CD44v8-10

60 causes suppression of phospho-p38 $8^{\mathrm{MAPK}}$, a major ROS target expression in transforming bile duct

61 cells which is linked to a poor prognosis in CCA patients. In addition, the regulation of redox

62 status in CCA cell lines depends on the expression of CD44v8-10 (Thanee et al. 2016).

63 Therefore, CD44v8-10 plays an important role in redox status regulation via stabilizing $\mathrm{xCT}$ in 
64 CCA development. During CCA genesis, oxidative stress during chronic inflammation may

65 induce gene related redox status regulation including CD44v to protect bile duct epithelial cells

66 from ROS, hence facilitating CCA genesis.

67 Additionally, iron is an essential nutrient and the most abundant transition metal in human

68 body, where under physiological conditions, it exists in its stable redox states, ferrous ion $\left(\mathrm{Fe}^{2+}\right)$

69 and ferric ion $\left(\mathrm{Fe}^{3+}\right.$ ) (Domaille et al. 2008). The essential roles of iron in various biological

70 events, such as oxygen delivery (Wilson \& Reeder 2008), electron transport (Rouault \& Tong

71 2005), and enzymatic reactions (Aisen et al. 1999; Costas et al. 2004), depend on its redox

72 activity. On the other hand, iron overload causes severe cell damage and organ dysfunction

73 through the abnormal production of ROS (Halliwell \& Gutteridge 1992; Xu et al. 2012). Thus,

74 iron plays critically important roles in both healthy and diseased states of the living organisms.

75 The role of iron in carcinogenesis has been shown to be associated with oxidative DNA damage

76 (Wiseman \& Halliwell 1996). We have previously reported that transferrin receptor-1 (TfR-1), a

77 cell surface receptor is a candidate molecule for involvement in the increasing of cellular iron

78 uptake in CCA (Jamnongkan et al. 2017). In addition, an increase in TfR-1 has also shown to be

79 responsible for transferrin-mediated iron uptake occurs in breast (Pinnix et al. 2010) and

80 pancreatic cancers (Ryschich et al. 2004).

81 Generally, the gold standard treatment for CCA is surgical resection. However, complete

82 resection is often impossible, and eventually when surgery is achieved, is typically followed by

83 metastasis and/or local recurrence (Khuntikeo et al. 2014; Titapun et al. 2015). The other

84 treatment options such as chemotherapy and radiotherapy have been ineffective for the patients

85 with inoperable tumors. Recently, extensive research has attempted to identify the effective

86 nontoxic nutrients, phytochemical and synthetic pharmacological agents which are believed to

87 have the ability to delay the onset of carcinogenesis process. Curcumin, an anti- inflammatory

88 agent, has been reported in many studies as a potential chemopreventive agent in CCA but the 
89 results are quite controversial. So far, it has been shown to inhibit an inflammatory reaction in Ov

90 infected hamsters, but it cannot inhibit Ov-induced CCA in the hamster animal model

91 (Boonjaraspinyo et al. 2009; Pinlaor et al. 2010; Prakobwong et al. 2009). Therefore, other

92 chemopreventive agents need to be identified and explored for their effectiveness in CCA

93 prevention and/or treatment.

94 Xanthohumol (XN) is a principal flavonoid of hop plant (Humulus lupulus L.) which has

95 been isolated from hop cones, that are largely used in the brewing industry as a preservative and

96 flavoring agent to add bitterness and aroma to beer (De Keukeleire et al. 1999; Taylor et al.

97 2003). It has been identified as having anti-inflammation, anti-oxidant and cancer

98 chemopreventive activities (Gerhauser 2005). Moreover, XN has been shown to be associated

99 with induction of apoptosis via increased p21 and p53 expression and decreased surviving levels

100 in a leukemia cell line (Monteghirfo et al. 2008). Additionally, it reduced ROS formation

101 (Hartkorn et al. 2009) and protection against DNA damage (Pinto et al. 2012). Dokduang et al.

102 reported that $\mathrm{XN}$ effectively suppressed the growth of tumor and induced apoptosis in CCA cells

103 and tumor inoculated mice by which inhibiting STAT3 activation due to suppression of the Akt-

104 NFאB signaling pathway (Dokduang et al. 2016). These data suggested that $\mathrm{XN}$ has a potential to

105 become a useful new approach for the chemoprevention and/or treatment of CCA. Additionally,

106 praziquantel (PZ) is well known as a potential drug for liver fluke treatment, which has also been

107 used for removal of Ov. The cure rate of PZ treatment in opisthorchiasis may be as high 100\%

108 effective in both humans (Bunnag \& Harinasuta 1981; Bunnag et al. 1984) and hamsters

109 (Duenngai et al. 2013). Decreasing levels of liver fibrosis and egg granulomas have been

110 revealed in the liver of patients with blood and liver fluke infections after PZ treatment (Pinlaor

111 et al. 2010).

112 Therefore, this study investigated the effect of XN with or without PZ, on DNA damage,

113 redox status change and periductal fibrosis during CCA genesis in a hamster animal model. 


\section{MATERIALS AND METHODS}

\section{Parasite preparation and animal infection}

116 Ov metacercariae were extracted from naturally infected cyprinid fish, which were

117 purchased from the market in Khon Kaen Province, northeast Thailand. The fish were minced

118 and digested with pepsin-HCL, then incubated in shaking water bath at $37^{\circ} \mathrm{C}$ for an hour. The

119 digested fish were filtered through the sieves (1,000, 425 and $106 \mu \mathrm{M}$ respectively) and the

120 filtrate was sedimented with $0.85 \%$ saline in a sedimentation jar. Finally, the metacercariae were

121 isolated and identified under a stereomicroscope. Fifty viable active cysts were fed to each of five

122 male Syrian golden hamsters by intragastric intubation.

\section{Xanthohumol preparation}

XN was kindly provided by Hopsteiner, Mainberge, Germany. XN -supplemented water

125 was prepared daily as $1 \mu \mathrm{l}$ of stock $20 \mathrm{mM}$ in $250 \mu \mathrm{l}$ of distilled-water, yielding a final

126 concentration of $20 \mu \mathrm{M}$ or $171 \mathrm{mg} / \mathrm{B} . \mathrm{W} . /$ day in the assigned groups. Hamsters were pre-treated

127 with XN more than 14 days before the experiment commenced and treatment was continued until

128 animals were sacrificed at day $60,90,120$ and 180 post-treatment.

$129 N$-nitrosodimethylamine preparation and administration

$130 N$-nitrosodimethylamine (NDMA) (Sigma-Aldrich, St. Louis MO, USA) was diluted in

131 distilled water at $12.5 \mathrm{ppm}$, and administrated daily to the assigned hamster groups starting on 132 day 30 until day 60 after Ov infection.

\section{Praziquantel preparation}

134 PZ (Sigma-Aldrich, St. Louis MO, USA) was diluted with 2\% chemophor, a non-ionic

135 solubilizer and emulsifier. A single dose of $400 \mathrm{mg} / \mathrm{kg}$ was administered orally to the assigned

136 treatment groups after day 30 of Ov infection.

\section{Animal groups}


139 study protocol. Six- to eight-week-old male Syrian golden hamsters were randomly divided into

140 four groups as followings: group I, Ov infection and NDMA administration (ON); group II, Ov

141 infection and NDMA administration and PZ treatment (ONP); groups III and IV were similar to

142 group I and II, they received $20 \mu \mathrm{M} \mathrm{XN}$ (171 mg/B.W./day) designated as XON and XONP

143 groups, respectively. Hamsters were treated with $50 \mathrm{Ov}$ metacercaria by oral inoculation which

144 was administered with $12.5 \mathrm{ppm}$ of NDMA in water for 30 days and withdrawn thereafter. XN-

145 supplemented water was prepared daily as $1 \mu 1$ of stock $20 \mathrm{mM}$ in $250 \mu 1$ of distilled-water. The

146 animals were sacrificed at 60, 90, 120 and 180 days after treatment and hamster liver tissues were

147 collected for further analysis.

148 General observations

149 Body and liver weights of each hamster were evaluated at the scarification time. Data

150 were expressed as mean \pm SD of liver/body weight. In addition, hamsters in $\mathrm{ON}, \mathrm{XON}, \mathrm{ONP}$, and

151 XONP groups were used for survival analysis, and the number of surviving hamsters was counted 152 monthly.

\section{Gross observation}

154 Four lobes of the liver (right, left, caudate, and quadrate) were examined for changes in

155 color, appearance of the margins, presence or absence of nodules, and granularity of surfaces,

156 both visually and from photographs taken with a digital camera.

157 Histological observation by Hematoxylin and Eosin (H\&E) staining

158 Hamster liver tissues were sectioned at a thickness of $4 \mu \mathrm{m}$, then fixed in $10 \%$ buffered 159 formaldehyde and embedded in paraffin. H\&E staining was performed in the sections for

160 histological observation. The sections of liver tissue were deparaffinized in xylene for 3 min for 161 three times to remove the paraffin wax, and then rehydrated in a dilution series of ethanol. The 162 sections were rinsed with tap water, stained with Harr's hematoxylin for 10 min, and then washed 
163 with the running tap water for 2 min. Destaining in ethanol containing $1 \%$ hydrochloric acid was

164 performed, then washed with running tap water, and stained in saturated lithium carbonate for 3-4

165 sec. The sections mounted on slides were stained with eosin solution after washing with running

166 tap water for 10-20 min. Dehydration of all sections was performed before mounting with

167 permount. Transformation of bile duct cells including normal, hyperplasia, dysplasia and

168 cholangiofibrosis, and cancerous lesions were examined under a light microscope (Axioscope A1,

169 Carl Zeiss, Jena, Germany) at high magnification (x200).

170 Immunohistochemistry

171 Sections mounted on slides were deparaffinized in xylene and rehydrated in an ethanol

172 concentration series. Three different methods were used to retrieve antigen from tissue sections;

173 1) for CD44 variant 8-10 staining; $0.5 \%$ trypsin-EDTA for $20 \mathrm{~min}$ at $37^{\circ} \mathrm{C}$ incubator, 2) for

174 phospho-p38 $8^{\mathrm{MAPK}}$ and 8-oxodG staining, 0.01 M sodium citrate containing $0.05 \%$ Tween 20 (pH

175 6.0) was autoclaved for $10 \mathrm{~min}$ at $110^{\circ} \mathrm{C}$, and 3) for TfR-1 microwave treatment was performed

176 in $10 \mathrm{mM}$ citrate buffer $\mathrm{pH} 6.0$ at high power for 10 minutes. After that, exposure of $3 \% \mathrm{H}_{2} \mathrm{O}_{2}$

177 and 3\% bovine serum albumin was performed before further incubation with primary and

178 secondary antibodies, respectively. A Vectastain Elite Kit (Vector Laboratories, Burlingame, CA)

179 and 3,3'-diaminobenzidine was used for detecting the staining signal, and the sections were

180 counterstained with Mayer's hematoxylin. The stained sections were observed under a light

181 microscope at high magnification $(\mathrm{x} 400)$. The intensity of staining was evaluated semi-

182 quantitatively by the Allred Scoring protocol (Allred et al. 1998).

\section{Prussian blue staining}

184 Prussian blue was used for localization of ferric iron in hamster liver tissues. Hamster

185 liver sections were deparaffinized and rehydrated with graded alcohol. Slides were immediately

186 transferred into a working staining solution (equal volumes of 10\% potassium ferrocyanide and 
$18710 \%$ hydrochloric acid) for 20 minutes at room temperature. Any ferric ions $\left(\mathrm{Fe}^{3+}\right)$ in the tissue

188 combines with the ferrocyanide and result in the formation of a bright blue pigment.

189 Subsequently, slides were rinsed in distilled water, and then counterstained with nuclear-fast red.

190 Finally, sections were dehydrated with stepwise increasing concentrations of ethanol, cleared

191 with xylene and mounted with permount. The stained sections were examined under a light

192 microscope.

193 Sirius red staining

194 Sirius red was used for collagen staining. Paraffin sections of each hamster group were 195 dewaxed in xylene and rehydrated with graded alcohol. The sections were stained with 196 haematoxylin and eosin for 8 minutes, washed in distilled water, incubated with $0.1 \%$ Sirius red 197 solution dissolved in aqueous saturated picric acid for 1 hour at room temperature, washed in 198 acidified water, dehydrated in xylene, and mounted with mounting medium. The slides were 199 analyzed by light microscope. Collagen, which is the component of fibrosis formation, stained 200 red around the bile duct areas. Therefore, the thickness of periductal fibrosis is stained in red 201 color. The evaluation of staining was adapted using the Batts-Ludwig and IASL for grading 202 fibrosis (Zachary D.G., 2007). Stage 0: no fibrosis; stage 1: mild fibrosis; stage 2: severe fibrosis. 203 Statistical analysis

204 Statistical analysis was performed using SPSS software. The difference in general 205 observation results was performed by student's $t$-test (nonparametric: Mann-Whitney test) in two206 tailed test. $P$-values of $<0.05$ were considered as statistically significant.

207 RESULTS

208 General observations

209 The liver per body weight of hamster groups either with or without XN and PZ were not 210 significantly different between groups. Moreover, survival curve analysis showed no statistical 
211 differences in the time that hamsters survived between each group. However, it seems that the

212 groups with $\mathrm{XN}$ treatment had longer survival times either in the presence or absence PZ

213 treatment but this was not statistical significant.

\section{Gross and histological appearances}

215 Gross pathology of the livers revealed smoothness of liver surfaces, although slightly

216 opaque around common bile ducts in all hamsters of both ON and XON groups throughout the

217 experimental period. White granules and small foci were found in the hepatic tissues at day 180

218 after treatment in both $\mathrm{ON}$ and XON groups (Figure 1). In addition, the obstruction of gall

219 bladder was seen in ON and XON groups but not in ONP and XONP groups. However, no tumor

220 mass was detected in the liver of all hamsters belonging to the above treatment groups.

221

Histopathological findings revealed no changes in livers and bile ducts in the XN

222 treatment alone, that was similar to untreated group, hence $\mathrm{XN}$ was not toxic to liver and bile

223 duct cells. In the ON group, aggregation of inflammatory cells, dilated bile ducts as well as

224 fibrosis was observed at all-time points. Additionally, massive hyperplasia and dysplasia of bile

225 ducts was observed at day 60, 90, 120 and 180. One of the hamsters $(1 / 5,20 \%)$ developed CCA

226 by day 180, whereas the others did not develop CCA. The most severe changes found in the rest

227 of hamsters at day 180 were cholangio-fibrosis. Interestingly, there was no CCA development

228 observed in the XON group. The most severe pathological changes observed at day 180 in this

229 group was cholangio-fibrosis $(4 / 5,80 \%)$, while only bile duct proliferation and hyperplasia were

230 seen in one of the hamster $(1 / 5,20 \%)$ as shown in Figure 2. These results indicated that XN

231 could repress the progression of CCA development. Importantly, there was no CCA development

232 in either PZ treated CCA-induced hamster (ONP) or combination with XN treatment (XONP).

233 The most severe pathological changes observed in these groups at day 180 was only bile duct

234 hyperplasia.

235 Effect of $\mathrm{XN}$ with or without PZ supplements on DNA damage 
237 the liver of hamsters was evaluated as detected by immunohistochemical staining. The expression

238 of 8-oxodG was observed mainly in the nucleus of bile ducts and inflammatory cells from day 60

239 and reached the highest on day 180 in ON group (Figure 3A). Interestingly, either PZ or XN

240 treatment significantly reduced the accumulation of 8-oxodG starting from day 60 and 120,

241 respectively, when compared with the ON group $(P<0.05)$. Moreover, our results showed that the

242 XONP group had significantly and more effectively reduced DNA damage than other groups

$243(P<0.05)$ (Figure 3B).

244 Effect of XN with or without PZ supplements on redox status

245 Our result showed that higher CD44v8-10 expression was seen along

246 cholangiocarcinogenesis. The strongest signal of CD44v8-10 was found at day 180 in both

247 dysplasia and malignant area of the bile duct epithelia in ON group (Figure 4A). On the other

248 hand, the highest signal of phospho-p38 $8^{\mathrm{MAPK}}$ which is a downstream target of ROS signaling was

249 seen in the nucleus of the hyperplastic bile duct cells at day 60 and then decreasing of phospho-

$250 \mathrm{p} 38^{\mathrm{MAPK}}$ level was seen along the progression of carcinogenesis in ON group (Figure 4C).

251 Furthermore, both protein levels of CD44v8-10 and phospho-p38 ${ }^{\mathrm{MAPK}}$ were significantly reduced

252 from day 60 in the ON group treated with either XN or PZ (XON and ONP) $(P<0.05)$ (Figure 4B

253 and D). However, no synergistic effect was observed in the XONP group.

254 Effect of XN with or without PZ supplements on iron accumulation and TfR-1 expression

255 Prussian blue was used for localization of ferric iron in hamster liver tissues. In the ON

256 group, iron accumulation was observed from 60 days until tumor developed at 180 days (Figure

257 5). The highest accumulation of iron was found at day 180 in both malignant bile duct epithelia

258 and inflammatory cells. In addition, immunohistrochemical staining for TfR-1 protein showed

259 that TfR-1 was localized in bile duct membranes and inflammatory cells (Figure 6A). The highest

260 of its expression was observed in cancerous lesions at day180 in ON group. Interestingly, the 
261 reduction of both molecules was seen in XON and ONP groups at day 90 when compared with

262 the ON group. Moreover, our results showed an effective reduction of iron accumulation and

263 TfR-1 expression for the XONP group compared to all other groups. This was especially evident

264 in the expression of TfR-1 in XONP group which was significantly reduced from day $90(\mathrm{P}<0.05)$

265 (Figure 6B).

266 The inhibitory effects of $\mathrm{XN}$ and PZ on fibrosis

267 The thickness of periductal fibrosis stained in red color is representative of severe or mild

268 fibrosis. In the ON group, severe fibrosis was seen starting from day 60 until day 180.

269 Additionally, treatment with either $\mathrm{XN}$ or PZ (XON and ONP groups) resulted in only mild

270 fibrosis at days 180 and 120, respectively. This was especially so, in the combination treatment or

271 XONP group, where only mild fibrosis was detected from day 60 as shown in Figure 7.

\section{DISCUSSION}

273 At present, the strategies for CCA prevention and treatment are still ineffective and

274 identifying new targets for potential therapeutic prevention of carcinogenesis should be

275 undertaken. A broad-spectrum of cancer chemopreventive agents which act by inhibiting multiple

276 mechanisms for cancer development has been reported (Gerhauser et al. 2002). One of the

277 interesting natural products for cancer prevention is XN. Many studies have indicated that XN

278 can prevent hepatic inflammation and fibrosis during chronic liver diseases as well as the

279 progression of liver cancer (Dorn et al. 2012). Additionally, XN could reduce ROS formation

280 (Hartkorn et al. 2009), protection against DNA damage (Pinto et al. 2012) and regulation of

281 apoptosis and suppression of hepatic stellate cell activation (Yang et al. 2013). These data

282 suggested that the $\mathrm{XN}$ has the potential to become a useful new approach for the

283 chemoprevention and/or treatment of CCA. 
285 CCA-induced hamster animal model. The expression of 8-oxodG which is an oxidative stress

286 marker was decreased in XN-treated groups. When used together with PZ (XONP) group it was

287 found to be more effective in reducing DNA damage leading to alteration of redox status and

288 repression of CCA development. This result correlated with our gross and histological

289 observations at day 180 of treatment, which demonstrated that $20 \%$ of ON group developed CCA

290 while in the remaing hamsters $(80 \%)$ only cholangio-fibrosis was found. After XN was

291 supplemented, $20 \%$ of hamsters had slow progression of CCA development because only bile

292 duct proliferation and hyperplasia were seen in these groups. Importantly, there was no CCA

293 development in combination with the PZ treatment (XONP). The most severe pathological

294 changes observed in these groups were only bile duct hyperplasia.

295 We then examined the effect of XN on alteration of redox status. Our results showed that

296 the redox status markers (CD44v8-10 and phospho-p38 $\left.{ }^{\mathrm{MAPK}}\right)$ were reduced in $\mathrm{XN}$ treatment

297 group, and may lead to repression of CCA development via anti-oxidant activity. XN could

298 protect cholangiocytes from oxidative stress during carcinogenesis, consequently, CD44v8-10

299 expression was not induced and phospho-p $38^{\mathrm{MAPK}}$ was also decreased.

300 From our previous study, we found that the accumulation of CD44v8-10 led to the

301 suppression of phospho-p38 $8^{\mathrm{MAPK}}$ in transforming bile duct cells. The redox status regulation of

302 CCA cells depends on the expression of CD44v8-10 to contribute the $\mathrm{xCT}$ function and is a link

303 to the poor prognosis of patients (Thanee et al. 2016). Importantly, we found that a decrease of

304 CD44v8-10 positive staining was seen during the transformation of bile duct epithelial cells from

305 normal, hyperplasia, and dysplasia in XN treated hamster CCA model. In addition, a high

306 phospho-p38 $8^{\mathrm{MAPK}}$ positive signal staining was observed in both bile duct epithelial cells and

307 surrounding inflammatory cells which was inversely correlated with the transformation of bile

308 duct cells. These results suggested that XN could reduce expression of CD44v8-10 via the 
309 possible mechanism of oxidative damage inhibition (reduction of phospho-p38MAPK

310 activation). Furthermore, it has been shown that XN could effectively suppress the growth of

311 tumor and induced apoptosis in CCA cells and tumor inoculated mice (Dokduang et al. 2016).

312 Currently, it has been reported that the oxidative stress could induce Wnt activation (Yoshida \&

313 Saya 2014), and then triggered the transcription of CD44 (Ishimoto et al. 2010). Moreover, our

314 group also showed an upregulation of Wnt/ $\beta$-catenin signaling corresponded with the period of

315 cholangiocarcinogenesis (Yothaisong et al. 2014). Based on this information, the possible

316 mechanism is that the liver fluke infection-induced chronic inflammation causing oxidative stress

317 leads to upregulation of Wnt/ $\beta$-catenin signaling pathways and consequently increasing of CD44

318 expression, which may drive to CCA carcinogenesis. Hence, an inhibition of oxidative damage

319 using XN could reduce expression of CD44v8-10 possibly via Wnt signaling pathway.

320 We next elucidated the effect of XN supplement on iron accumulation and TfR-1

321 expression. We found both decreasing iron accumulation and TfR-1 expression in bile ducts and

322 inflammatory cells. Iron is an important determinant of ROS generation because an excess of iron

323 can undergo redox reaction and stimulate free radical formation. $\mathrm{H}_{2} \mathrm{O}_{2}$ - mediated toxicity was

324 found to be iron mediated. TfR-1 knockdown has been shown to reduce iron uptake by $80 \%$ in

325 human hepatoma cell line (Herbison et al. 2009). Moreover, the aberrant expression of TfR-1

326 substantially contributed to the regulation of systemic iron levels and can play key roles in CCA

327 development (Jamnongkan et al. 2017). TfR-1 has been reported to be a major molecule that

328 responded to iron uptake mediated by insulin through hypoxia conditions (Biswas et al. 2013).

329 Andriopoulos et al. found that exposure of cultured cells to sustained low levels of hydrogen

330 peroxide that mimic its release by inflammatory cells lead to up-regulation of TfR-1

331 (Andriopoulos et al. 2007). In steatotic livers, the saturation of $\beta$-oxidation by excess free fatty

332 acids will ultimately lead to the generation of hydrogen peroxide, which in turn can be converted

333 to highly reactive hydroxyl radicals in the presence of free iron via Fenton reaction (Videla et al. 
334 2003). Indeed, there is strong evidence, from both in vitro and in vivo studies, that iron overload

335 enhances oxidative stress (Brown et al. 2003; Cornejo et al. 2005; Kadiiska et al. 1995).

336 Sirius red staining was used in each hamster group to assess for levels of collagen which

337 is related to fibrosis. In the revealed a thick and extensive periductal fibrosis was found in the ON

338 group which could be related to Ov-induced fibrosis caused by bile duct lesions that

339 corresponded to the amount of collagen detected. The increased amount of collagen we detected

340 around bile ducts is similar to findings by Prakobwong et al. (2009) who also showed that it is

341 dependent on the duration of infection. In addition, we found that $\mathrm{XN}$ could reduce fibrosis from

342 day 90 to 180 in another group of hamsters (XON group) (Prakobwong et al. 2009). Hence, XN

343 has the potential to reduce periductal fibrosis in early stages of liver fluke infection as well as

344 efficiently reducing fibrosis in long time treatment. We found that the reduction of fibrosis due to

345 Ov infection was efficiently eliminated by PZ treatment in the ONP group. These results are

346 similar to those of Pinlaor et al. who showed the effectiveness of PZ treatment for complete

347 reduction of the fibrosis in liver tissues in order to prevent the progression of the parasite-induced

348 diseases, and CCA development (Pinlaor et al. 2009). Moreover, our study showed that the

349 greatest reduction of fibrosis was in the XONP group compared to all other groups.

350 Praziquantel is well known as a potential drug for Ov treatment and removal of Ov. The

351 cure rate of $\mathrm{PZ}$ treatment in opisthorchiasis can be as much as $100 \%$ effective in people (Bunnag

352 \& Harinasuta 1981; Bunnag et al. 1984) and hamster (Duenngai et al. 2013). Decreasing liver

353 fibrosis and egg granulomas has been revealed in the liver of patients with blood and liver fluke

354 infections after PZ treatment (Pinlaor et al. 2010). In addition, it was demonstrated that

355 promutagenic etheno-DNA and 8-oxodG adducts are most likely to increase the risk of Ov-

356 infected patients to later develop CCA. A relationship between these adduct markers and disease

357 causation is further supported by the protective effect of PZ against DNA damage in human

358 (Dechakhamphu et al. 2010; Thanan et al. 2008). Similarly, this study suggests that CCA 
359 development might be suppressed after Ov treatment with PZ. Moreover, we have observed a

360 high efficiency to suppress the progression of CCA in combination treatments between XN and

361 PZ (XONP group). Other studies have indicated that the combination of curcumin (Charoensuk et

362 al. 2011), and Thunbergia laurifolia (Wonkchalee et al. 2013) with PZ treatment improved the

363 hepatobiliary system and could retard CCA progression.

\section{CONCLUSIONS}

365 In conclusion, we have demonstrated that $\mathrm{PZ}$ can reduce chronic inflammation in

366 combination with $\mathrm{XN}$ treatment as well as suppressing oxidative stress possibly via the reduction

367 of DNA damage, iron accumulation, periductal fibrosis and the alterations of intracellular redox

368 lead to delayed CCA development. The proposed mechanism is shown in Figure8. Hence, $\mathrm{XN}$ in

369 combination with PZ may prove to be useful chemopreventive agents for CCA prevention.

\section{ACKNOWLEDEMENTS}

371 We would like to acknowledge Prof. Ross H Andrews for editing the MS via Publication

372 Clinic KKU, Thailand.

\section{REFERENCES}

374 Aisen P, Wessling-Resnick M, and Leibold EA. 1999. Iron metabolism. Curr Opin Chem Biol 3:200-206. 10.1016/S1367-5931(99)80033-7

Allred DC, Harvey JM, Berardo M, and Clark GM. 1998. Prognostic and predictive factors in breast cancer by immunohistochemical analysis. Mod Pathol 11:155-168.

378 Andriopoulos B, Hegedusch S, Mangin J, Riedel HD, Hebling U, Wang J, Pantopoulos K, and Mueller S. 2007. Sustained hydrogen peroxide induces iron uptake by transferrin 
receptor-1 independent of the iron regulatory protein/iron-responsive element network. $J$ Biol Chem 282:20301-20308. 10.1074/jbc.M702463200

382 Biswas S, Tapryal N, Mukherjee R, Kumar R, and Mukhopadhyay CK. 2013. Insulin promotes iron uptake in human hepatic cell by regulating transferrin receptor-1 transcription mediated by hypoxia inducible factor-1. Biochim Biophys Acta 1832:293-301.

Boonjaraspinyo S, Boonmars T, Aromdee C, Srisawangwong T, Kaewsamut B, Pinlaor S, Yongvanit P, and Puapairoj A. 2009. Turmeric reduces inflammatory cells in hamster opisthorchiasis. Parasitol Res 105:1459-1463.

Brown KE, Dennery PA, Ridnour LA, Fimmel CJ, Kladney RD, Brunt EM, and Spitz DR. 2003. Effect of iron overload and dietary fat on indices of oxidative stress and hepatic fibrogenesis in rats. Liver Int 23:232-242.

Bunnag D, and Harinasuta T. 1981. Studies on the chemotherapy of human opisthorchiasis: III. Minimum effective dose of praziquantel. Southeast Asian J Trop Med Public Health $12: 413-417$

Bunnag D, Pungpark S, Harinasuta T, Viravan C, Vanijanonta S, Suntharasamai P, Migasena S,

Chamadol N, Pairojkul C, Khuntikeo N, Laopaiboon V, Loilome W, Sithithaworn P, and experience with praziquantel in Hospital for Tropical Diseases. Arzneimittelforschung Yongvanit P. 2014. Histological confirmation of periductal fibrosis from ultrasound diagnosis in cholangiocarcinoma patients. J Hepatobiliary Pancreat Sci 21:316-322. $10.1002 /$ jhbp. 64

Charoensuk L, Pinlaor P, Prakobwong S, Hiraku Y, Laothong U, Ruangjirachuporn W, Yongvanit P, and Pinlaor S. 2011. Curcumin induces a nuclear factor-erythroid 2-related factor 2- 
driven response against oxidative and nitrative stress after praziquantel treatment in liver

406 fluke-infected hamsters. Int J Parasitol 41:615-626. 10.1016/j.ijpara.2010.12.011

407 Cornejo P, Varela P, Videla LA, and Fernandez V. 2005. Chronic iron overload enhances

408

409 inducible nitric oxide synthase expression in rat liver. Nitric Oxide 13:54-61. 10.1016/j.niox.2005.04.009

Costas M, Mehn MP, Jensen MP, and Que L, Jr. 2004. Dioxygen activation at mononuclear nonheme iron active sites: enzymes, models, and intermediates. Chem Rev 104:939-986.

413 De Keukeleire D, De Cooman L, Rong H, Heyerick A, Kalita J, and Milligan SR. 1999.

414 Functional properties of hop polyphenols. Basic Life Sci 66:739-760.

415 Dechakhamphu S, Pinlaor S, Sitthithaworn P, Nair J, Bartsch H, and Yongvanit P. 2010. Lipid peroxidation and etheno DNA adducts in white blood cells of liver fluke-infected patients: protection by plasma alpha-tocopherol and praziquantel. Cancer Epidemiol Biomarkers Prev 19:310-318. 10.1158/1055-9965.EPI-09-0849

Dokduang H, Yongvanit P, Namwat N, Pairojkul C, Sangkhamanon S, Yageta MS, Murakami Y, and Loilome W. 2016. Xanthohumol inhibits STAT3 activation pathway leading to growth

423 Domaille DW, Que EL, and Chang CJ. 2008. Synthetic fluorescent sensors for studying the cell biology of metals. Nat Chem Biol 4:168-175. 10.1038/nchembio.69

Dorn C, Heilmann J, and Hellerbrand C. 2012. Protective effect of xanthohumol on toxin-induced liver inflammation and fibrosis. Int J Clin Exp Pathol 5:29-36.

427 Duenngai K, Boonmars T, Sithithaworn J, and Sithithaworn P. 2013. Diagnosis of early infection and post chemotherapeutic treatment by copro-DNA detection in experimental opisthorchiasis. Parasitol Res 112:271-278. 10.1007/s00436-012-3134-0 
430 Gerhauser C. 2005. Beer constituents as potential cancer chemopreventive agents. Eur J Cancer

431

432

433

434

435

436

437

438

439

440

441

442

443

444

445

446

447

448

449

450

451

452

453

$$
\text { 41:1941-1954. }
$$

Gerhauser C, Alt A, Heiss E, Gamal-Eldeen A, Klimo K, Knauft J, Neumann I, Scherf HR, Frank N, Bartsch H, and Becker H. 2002. Cancer chemopreventive activity of Xanthohumol, a natural product derived from hop. Mol Cancer Ther 1:959-969.

Halliwell B, and Gutteridge JM. 1992. Biologically relevant metal ion-dependent hydroxyl radical generation. An update. FEBS Lett 307:108-112.

Hartkorn A, Hoffmann F, Ajamieh H, Vogel S, Heilmann J, Gerbes AL, Vollmar AM, and Zahler S. 2009. Antioxidant effects of xanthohumol and functional impact on hepatic ischemiareperfusion injury. J Nat Prod 72:1741-1747. 10.1021/np900230p

Herbison CE, Thorstensen K, Chua AC, Graham RM, Leedman P, Olynyk JK, and Trinder D. 2009. The role of transferrin receptor 1 and 2 in transferrin-bound iron uptake in human hepatoma cells. Am J Physiol Cell Physiol 297:C1567-1575. 10.1152/ajpcell.00649.2008

Ishimoto T, Nagano O, Yae T, Tamada M, Motohara T, Oshima H, Oshima M, Ikeda T, Asaba R, Yagi H, Masuko T, Shimizu T, Ishikawa T, Kai K, Takahashi E, Imamura Y, Baba Y, Ohmura M, Suematsu M, Baba H, and Saya H. 2011. CD44 variant regulates redox status in cancer cells by stabilizing the $\mathrm{xCT}$ subunit of system $\mathrm{xc}(-)$ and thereby promotes tumor growth. Cancer Cell 19:387-400. 10.1016/j.ccr.2011.01.038

Ishimoto T, Oshima H, Oshima M, Kai K, Torii R, Masuko T, Baba H, Saya H, and Nagano O. 2010. CD44+ slow-cycling tumor cell expansion is triggered by cooperative actions of Wnt and prostaglandin E2 in gastric tumorigenesis. Cancer Sci 101:673-678. 10.1111/j.1349-7006.2009.01430.x

Jamnongkan W, Thanan R, Techasen A, Namwat N, Loilome W, Intarawichian P, Titapun A, and Yongvanit P. 2017. Upregulation of transferrin receptor-1 induces cholangiocarcinoma 
progression via induction of labile iron pool. Tumour Biol 39:1010428317717655.

456 Kadiiska MB, Burkitt MJ, Xiang QH, and Mason RP. 1995. Iron supplementation generates hydroxyl radical in vivo. An ESR spin-trapping investigation. J Clin Invest 96:1653-1657. 10.1172/JCI118205

Khuntikeo N, Pugkhem A, Titapun A, and Bhudhisawasdi V. 2014. Surgical management of perihilar cholangiocarcinoma: a Khon Kaen experience. J Hepatobiliary Pancreat Sci

462 Kim GY, Mercer SE, Ewton DZ, Yan Z, Jin K, and Friedman E. 2002. The stress-activated protein kinases p38 alpha and JNK1 stabilize p21(Cip1) by phosphorylation. J Biol Chem

Mairiang E, Elkins DB, Mairiang P, Chaiyakum J, Chamadol N, Loapaiboon V, Posri S, Sithithaworn P, and Haswell-Elkins M. 1992. Relationship between intensity of Opisthorchis viverrini infection and hepatobiliary disease detected by ultrasonography. $J$

469 Monteghirfo S, Tosetti F, Ambrosini C, Stigliani S, Pozzi S, Frassoni F, Fassina G, Soverini S,

470 Albini A, and Ferrari N. 2008. Antileukemia effects of xanthohumol in Bcr/Abltransformed cells involve nuclear factor-kappaB and p53 modulation. Mol Cancer Ther

473 Pinlaor S, Prakobwong S, Boonmars T, Wongkham C, Pinlaor P, and Hiraku Y. 2009. Effect of 474 praziquantel treatment on the expression of matrix metalloproteinases in relation to tissue 475 resorption during fibrosis in hamsters with acute and chronic Opisthorchis viverrini infection. Acta Tropica 111:181-191. 10.1016/j.actatropica.2009.04.011 
477 Pinlaor S, Prakobwong S, Hiraku Y, Pinlaor P, Laothong U, and Yongvanit P. 2010. Reduction of

478 periductal fibrosis in liver fluke-infected hamsters after long-term curcumin treatment.

479 Eur J Pharmacol 638:134-141. 10.1016/j.ejphar.2010.04.018

480 Pinnix ZK, Miller LD, Wang W, D'Agostino R, Jr., Kute T, Willingham MC, Hatcher H, Tesfay L,

481 Sui G, Di X, Torti SV, and Torti FM. 2010. Ferroportin and iron regulation in breast

482 cancer progression and prognosis. Sci Transl Med 2:43ra56. 10.1126/scisignal.3001127

483 Pinto C, Duque AL, Rodriguez-Galdon B, Cestero JJ, and Macias P. 2012. Xanthohumol prevents carbon tetrachloride-induced acute liver injury in rats. Food Chem Toxicol 50:3405-3412. 10.1016/j.fct.2012.07.035

Prakobwong S, Pinlaor S, Yongvanit P, Sithithaworn P, Pairojkul C, and Hiraku Y. 2009. Time profiles of the expression of metalloproteinases, tissue inhibitors of metalloproteases, cytokines and collagens in hamsters infected with Opisthorchis viverrini with special reference to peribiliary fibrosis and liver injury. Int J Parasitol 39:825-835.

Rouault TA, and Tong WH. 2005. Iron-sulphur cluster biogenesis and mitochondrial iron homeostasis. Nat Rev Mol Cell Biol 6:345-351. 10.1038/nrm1620

Ryschich E, Huszty G, Knaebel HP, Hartel M, Buchler MW, and Schmidt J. 2004. Transferrin receptor is a marker of malignant phenotype in human pancreatic cancer and in neuroendocrine carcinoma of the pancreas. Eur J Cancer 40:1418-1422. proanthocyanidins characterized by mass spectrometry, acid catalysis, and gel permeation chromatography. J Agric Food Chem 51:4101-4110. patients with parasite infection and effect of antiparasitic drug in relation to 
504 Thanee M, Loilome W, Techasen A, Sugihara E, Okazaki S, Abe S, Ueda S, Masuko T, Namwat

505 N, Khuntikeo N, Titapun A, Pairojkul C, Saya H, and Yongvanit P. 2016. CD44 variant-

506 dependent redox status regulation in liver fluke-associated cholangiocarcinoma: A target

507 for cholangiocarcinoma treatment. Cancer Sci 107:991-1000. 10.1111/cas.12967

508 Titapun A, Pugkhem A, Luvira V, Srisuk T, Somintara O, Saeseow OT, Sripanuskul A,

509 Nimboriboonporn A, Thinkhamrop B, and Khuntikeo N. 2015. Outcome of curative

510 resection for perihilar cholangiocarcinoma in Northeast Thailand. World J Gastrointest

S11 Oncol 7:503-512. 10.4251/wjgo.v7.i12.503

512 Videla LA, Fernandez V, Tapia G, and Varela P. 2003. Oxidative stress-mediated hepatotoxicity

513 of iron and copper: role of Kupffer cells. Biometals 16:103-111.

514 Wilson MT, and Reeder BJ. 2008. Oxygen-binding haem proteins. Exp Physiol 93:128-132.

$515 \quad$ 10.1113/expphysiol.2007.039735

516 Wiseman H, and Halliwell B. 1996. Damage to DNA by reactive oxygen and nitrogen species:

517 role in inflammatory disease and progression to cancer. Biochem J $313(\mathrm{Pt}$ 1):17-29.

518 Wonkchalee N, Boonmars T, Laummaunwai P, Aromdee C, Hahnvajanawong C, Wu Z, Sriraj P, 519 Aukkanimart R, Chamgramol Y, Pairojkul C, Juasook A, and Sudsarn P. 2013. A

520 combination of praziquantel and the traditional medicinal plant Thunbergia laurifolia on

521 Opisthorchis viverrini infection and cholangiocarcinoma in a hamster model. Parasitol

$522 \quad \operatorname{Res} 112: 4211-4219.10 .1007 / \mathrm{s} 00436-013-3613-\mathrm{y}$

$523 \mathrm{Xu}$ J, Jia Z, Knutson MD, and Leeuwenburgh C. 2012. Impaired iron status in aging research. Int J Mol Sci 13:2368-2386. 10.3390/ijms13022368

525 Yang M, Li N, Li F, Zhu Q, Liu X, Han Q, Wang Y, Chen Y, Zeng X, Lv Y, Zhang P, Yang C, and 526 Liu Z. 2013. Xanthohumol, a main prenylated chalcone from hops, reduces liver damage 

belangeri. Int Immunopharmacol 16:466-474. 10.1016/j.intimp.2013.04.029

529 Yongvanit P, Pinlaor S, and Bartsch H. 2012a. Oxidative and nitrative DNA damage: key events $530 \quad$ in opisthorchiasis-induced carcinogenesis. Parasitol Int 61:130-135.

531 Yongvanit P, Thanan R, Pinlaor S, Sithithaworn P, Loilome W, Namwat N, Techasen A, and 532 Dechakhamphu S. 2012b. Increased expression of TLR-2, COX-2, and SOD-2 genes in 533 the peripheral blood leukocytes of opisthorchiasis patients induced by Opisthorchis 534 viverrini antigen. Parasitol Res 110:1969-1977. 10.1007/s00436-011-2725-5

535 Yoshida GJ, and Saya H. 2014. Inversed relationship between CD44 variant and c-Myc due to 536 oxidative stress-induced canonical Wnt activation. Biochem Biophys Res Commun $537 \quad 443: 622-627.10 .1016 /$ j.bbrc.2013.12.016

538 Yothaisong S, Thanee M, Namwat N, Yongvanit P, Boonmars T, Puapairoj A, and Loilome W. 539 2014. Opisthorchis viverrini infection activates the PI3K/AKT/PTEN and Wnt/beta540 catenin signaling pathways in a Cholangiocarcinogenesis model. Asian Pac J Cancer Prev 15:10463-10468. 


\section{Figure 1}

Gross findings of hamster liver tissues.

Photographs are representative at days $60,90,120$, and 180 in four groups including the control (ON), presence of xanthohumol (XON), presence of praziquantel (ONP) and presence of xanthohumol and praziquantel (XONP) groups. Results show the obstruction of gall bladder in ON and XON groups at $60,90,120$, and 180 days but not in the ONP and XONP groups. Additionally, white granules were observed at day 180 in ON and XON groups. Arrowheads indicate white granules and small foci.
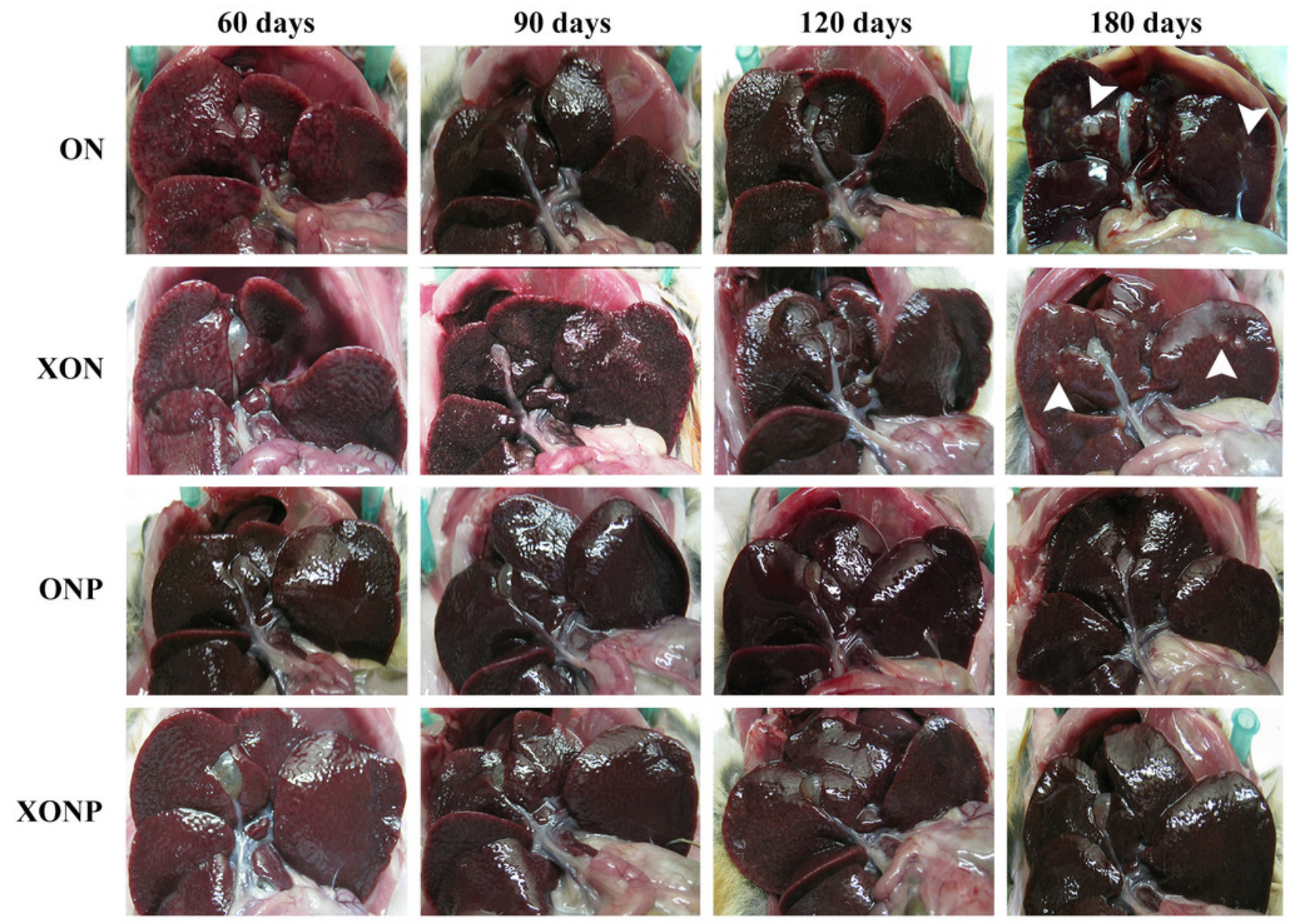


\section{Figure 2}

Histological changes in Ov-induced CCA hamsters with the control (ON), presence of xanthohumol (XON), presence of praziquantel (ONP) and presence of xanthohumol and praziquantel (XONP) groups.

Circles represent: Ov, O. viverrini; BD, bile duct; IF, inflammation area; HD, hyperplasia bile duct; PD, proliferative bile duct.

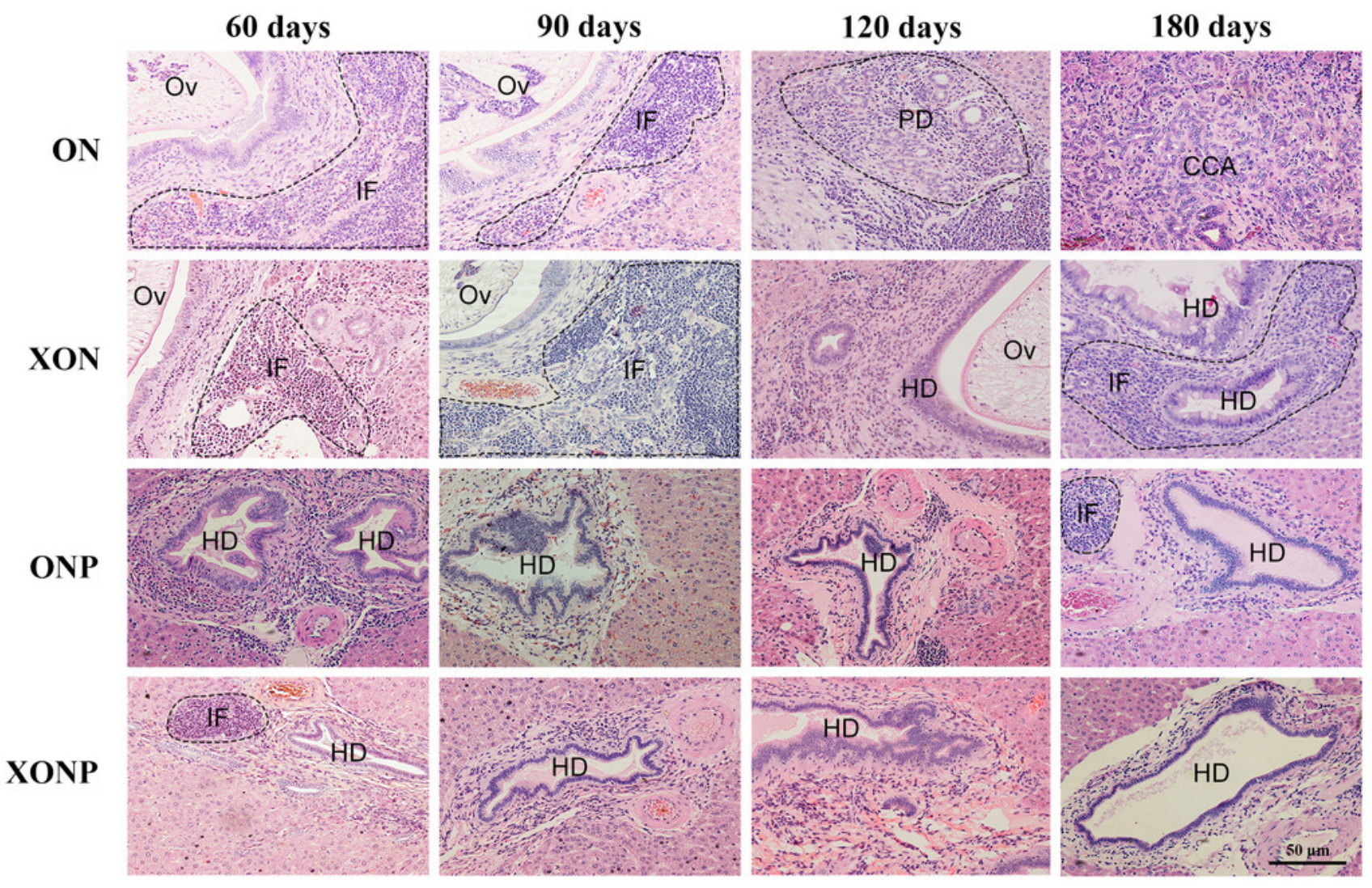




\section{Figure 3}

Immunostaining of 8-oxodG in CCA genesis hamsters.

Localization of 8-oxodG in the control (ON), presence of xanthohumol (XON), presence of praziquantel (ONP) and presence of xanthohumol and praziquantel (XONP) groups (A). Bar graph comparisons of frequency and intensity using Allred score in each group of hamster liver (B). $* P<0.05$ compared to ON group. 


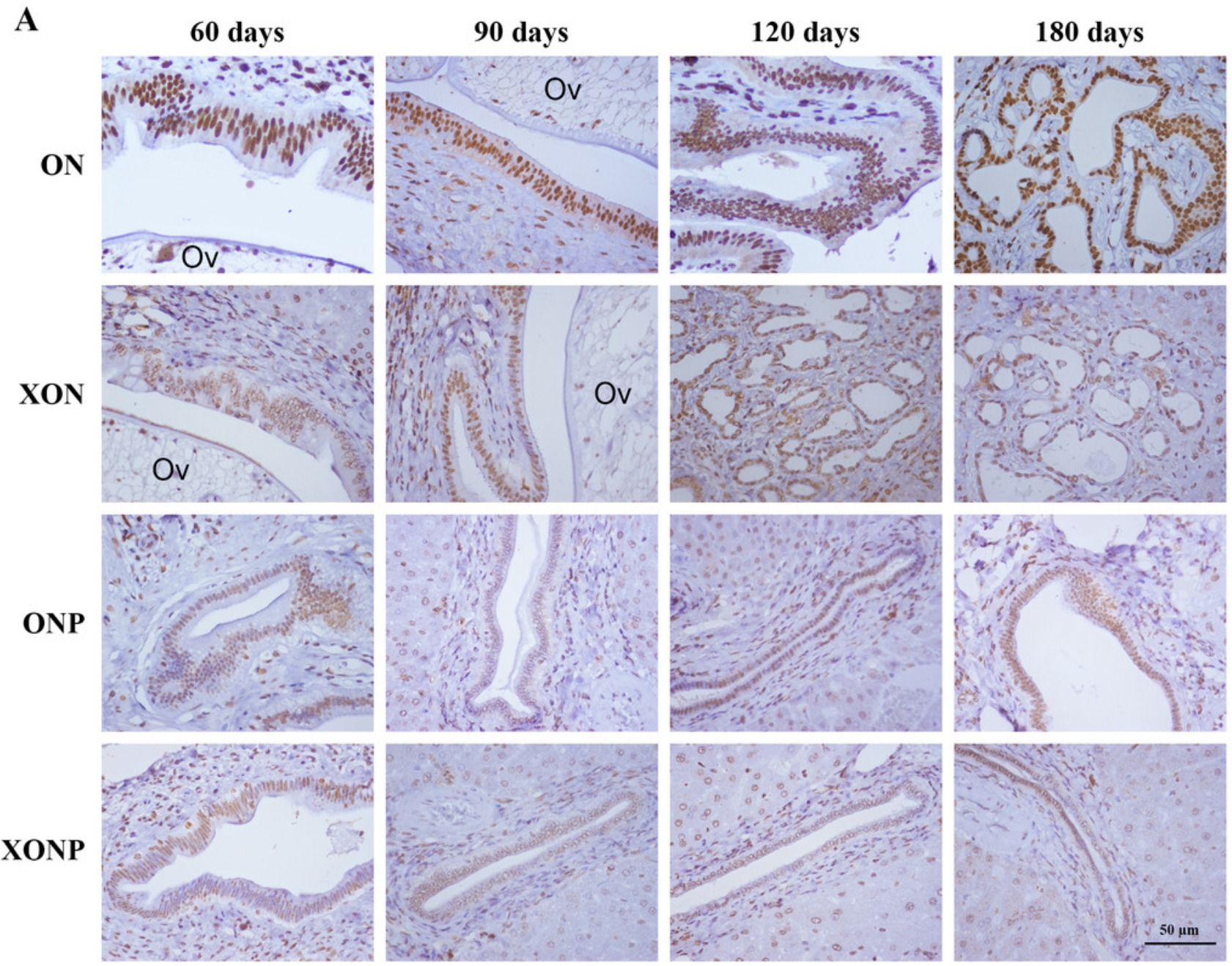

B

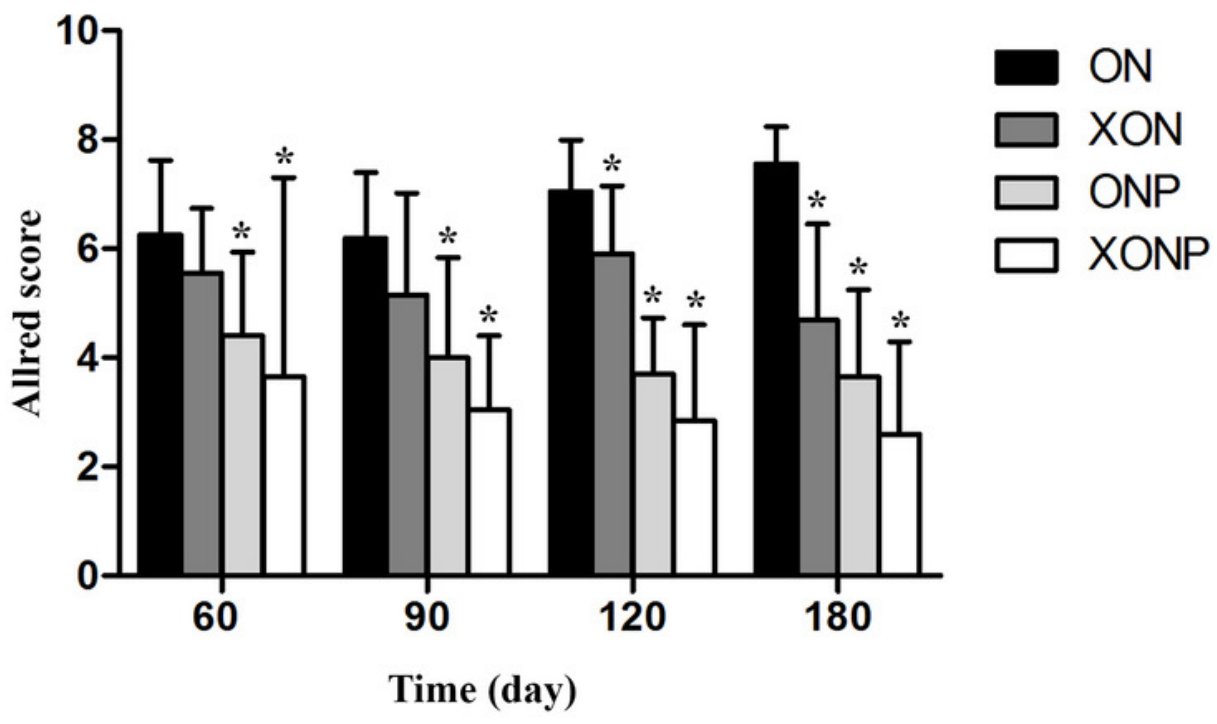




\section{Figure 4}

Immunostaining of CD44v8-10; cancer stem-like cells $(A)$ and phospho-p38 ${ }^{\text {MAPK}}$; downstream of ROS signaling $(C)$ in CCA genesis in hamsters.

Photographs are representative of the control (ON), presence of xanthohumol (XON), presence of praziquantel (ONP) and presence of xanthohumol and praziquantel (XONP) groups. Bar graph comparisons of frequency and intensity using Allred score in each group of hamster liver (B and D). $* P<0.05$ compared to ON group. 
A
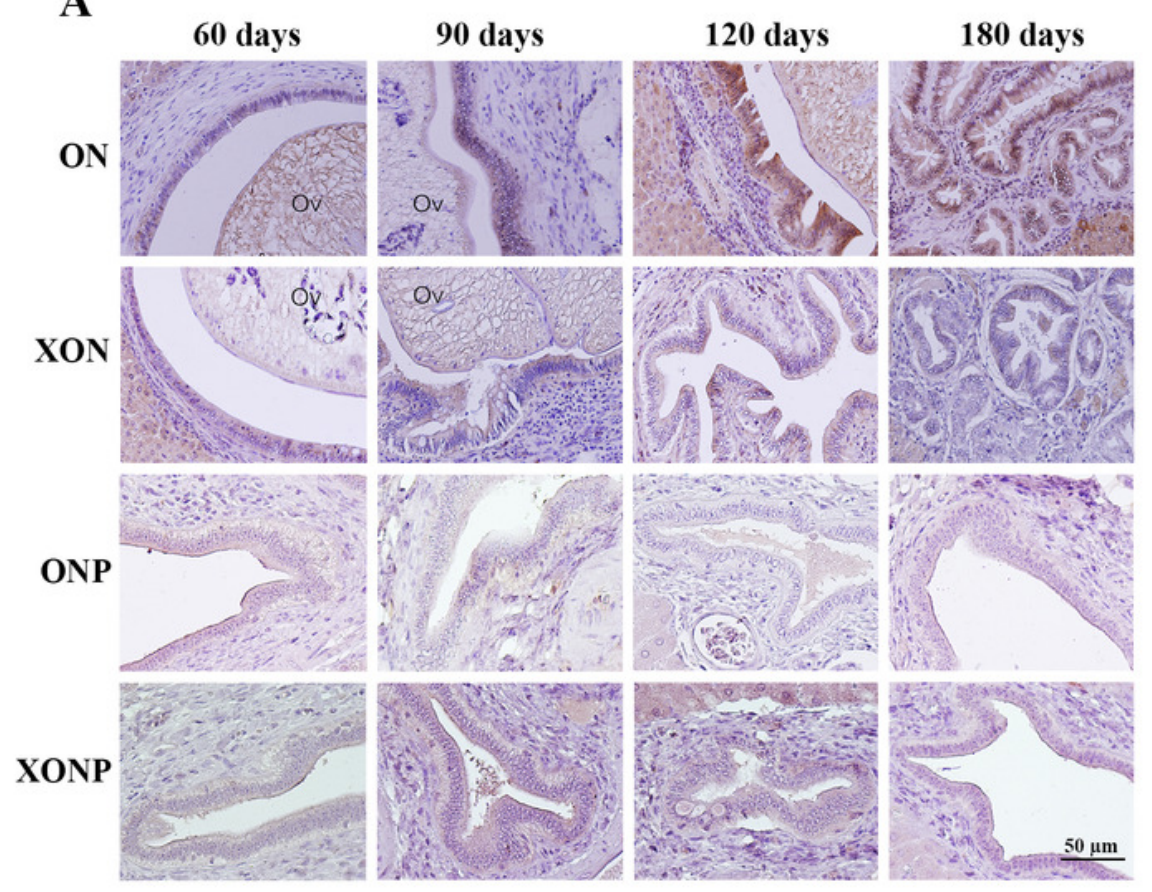

C

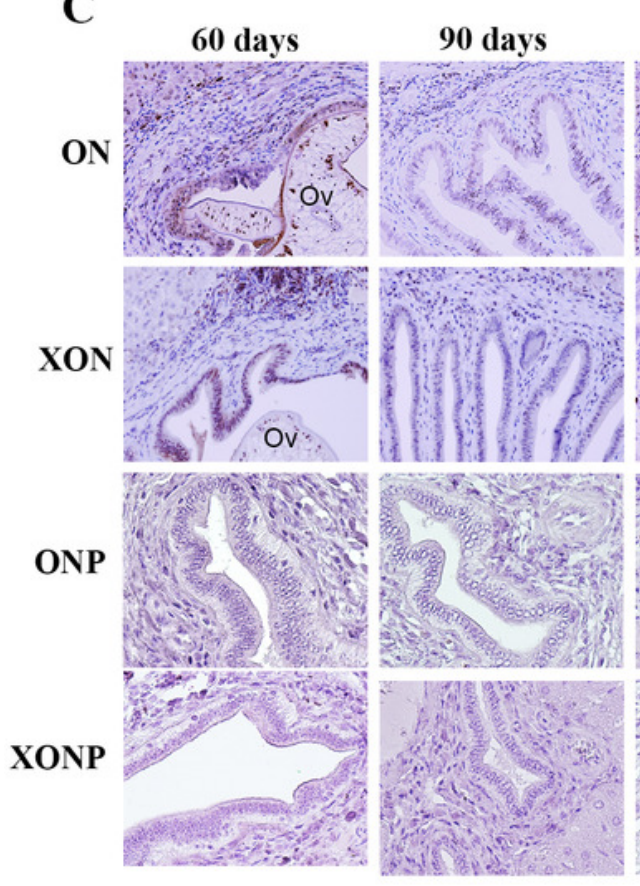

120 days
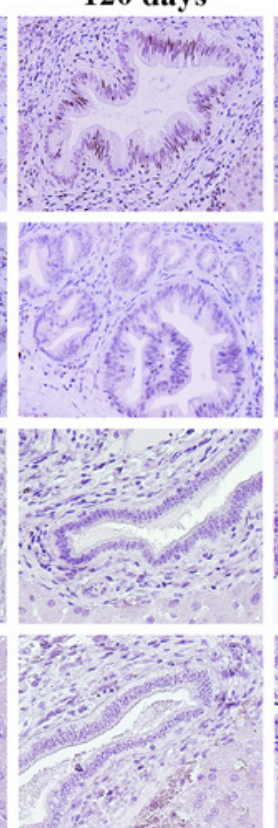

180 days
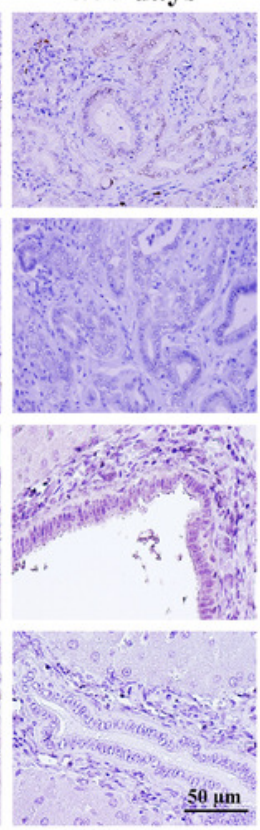

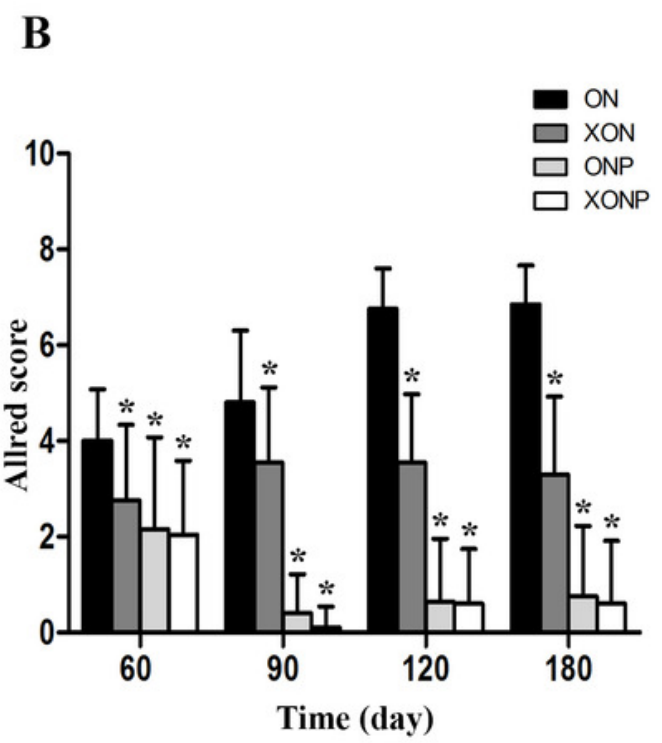

D

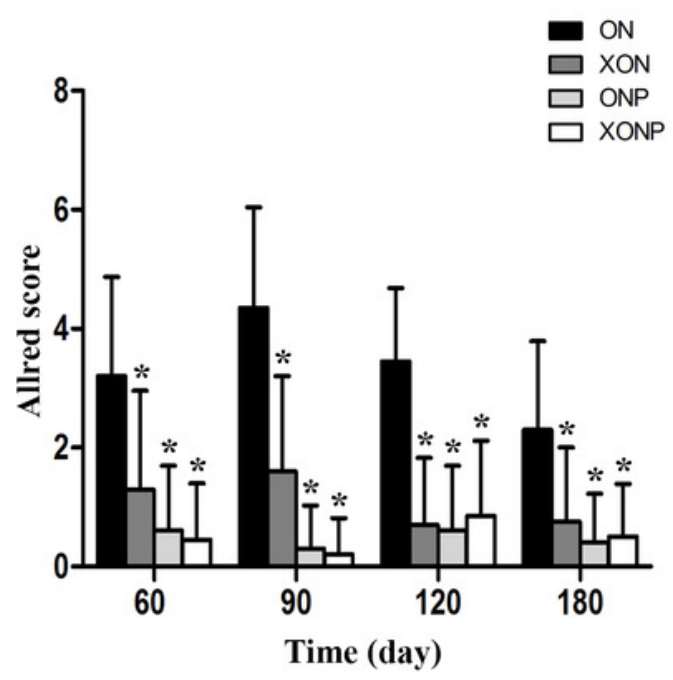




\section{Figure 5}

Prussian blue staining of iron in CCA genesis hamsters.

Photographs are representative of the control (ON), presence of xanthohumol (XON), presence of praziquantel (ONP) and presence of xanthohumol and praziquantel (XONP) groups.

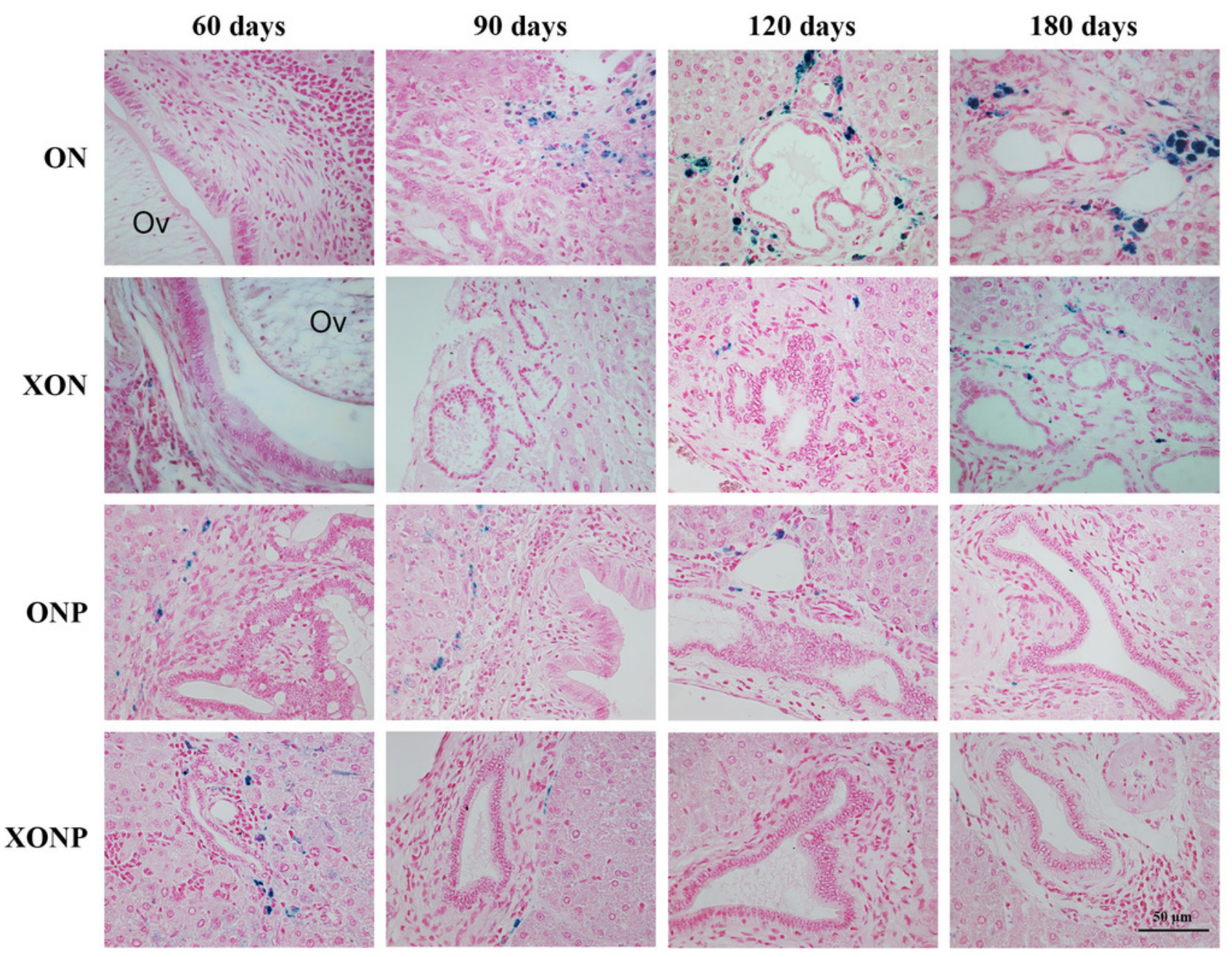


Figure 6

Immunohistochemistry for in CCA genesis hamsters.

Photographs are representative of the control (ON), presence of xanthohumol (XON), presence of praziquantel (ONP) and presence of xanthohumol and praziquantel (XONP) groups (A). Bar graph comparisons of frequency and intensity using Allred score in each group of hamster liver (B). $* P<0.05$ compared to ON group.

A

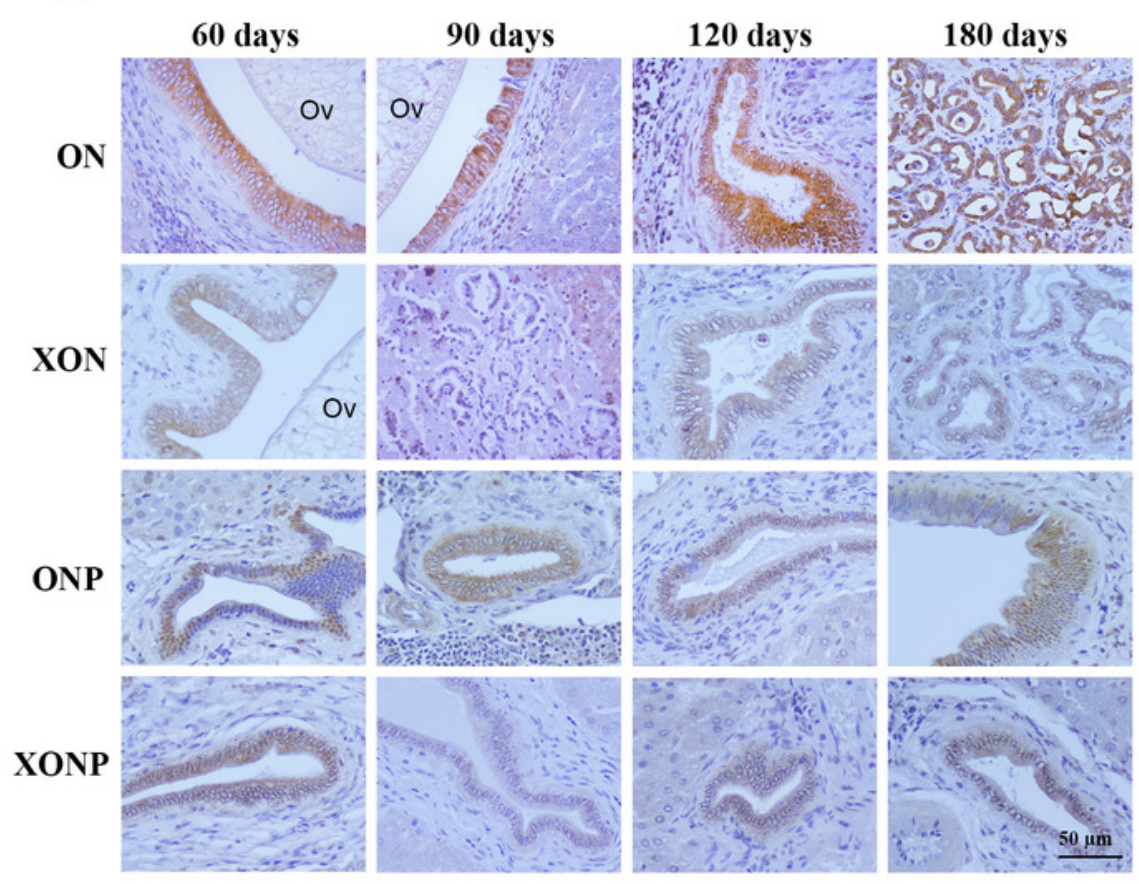

B

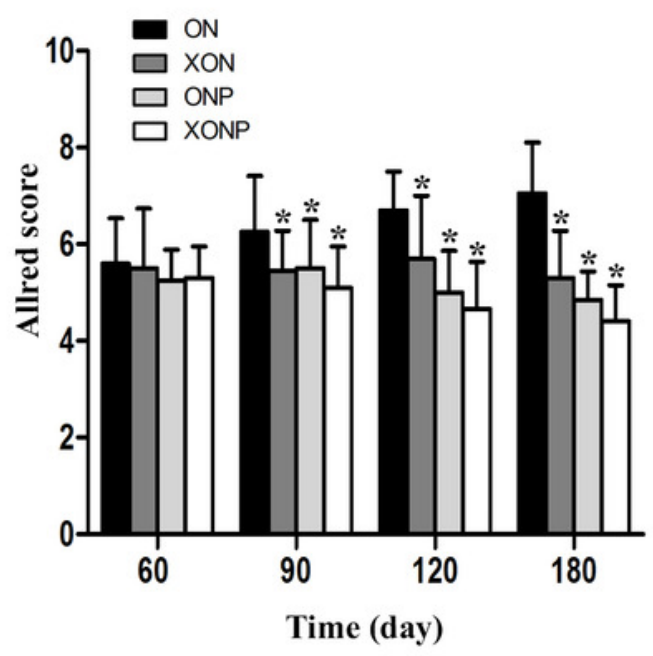




\section{Figure 7}

Sirius red staining for collagen in CCA genesis hamsters with the control (ON), presence of xanthohumol (XON), presence of praziquantel (ONP) and presence of xanthohumol and praziquantel (XONP) groups.

Thickness of red color represents fibrosis area.

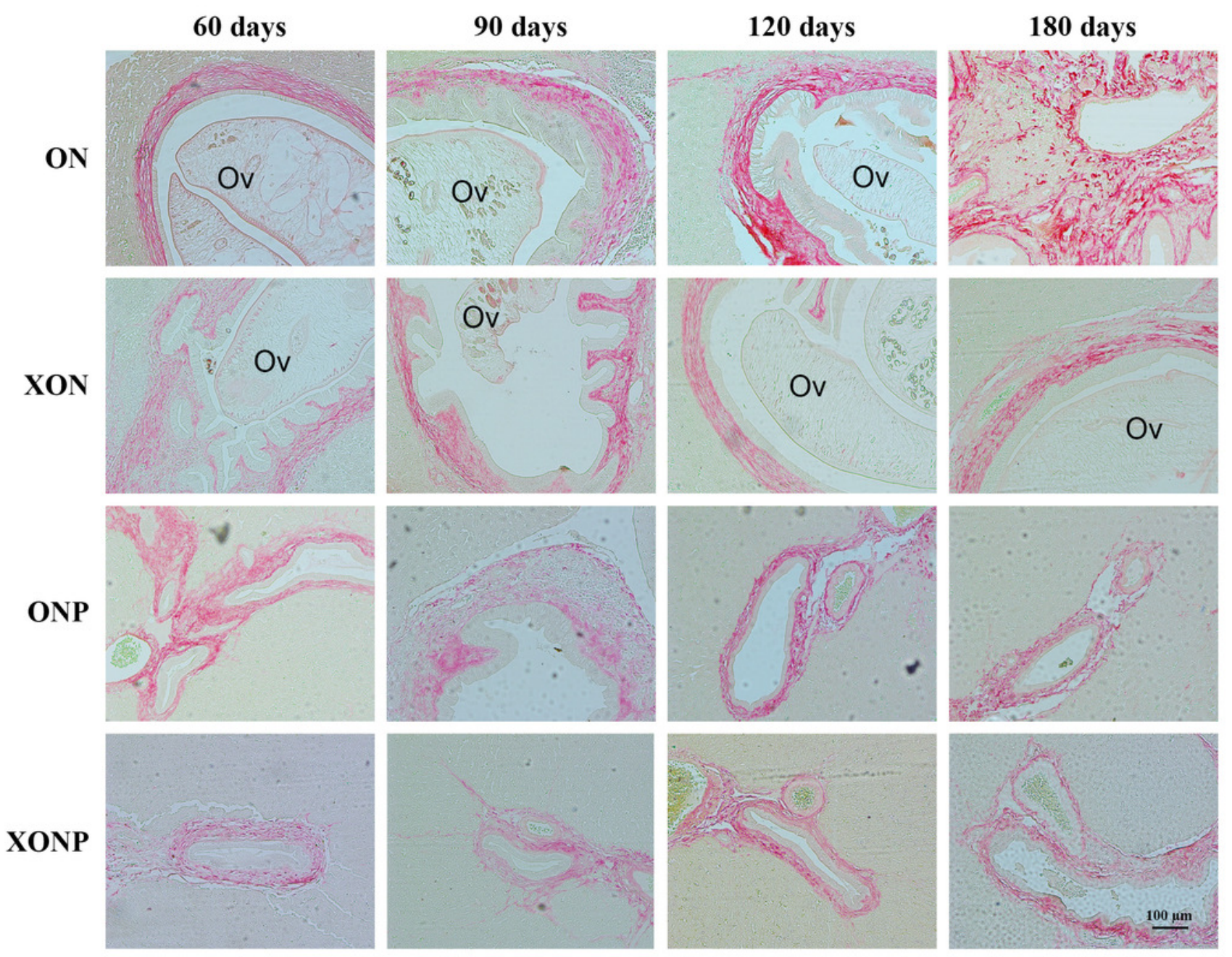




\section{Figure 8 (on next page)}

A possible chemopreventive effect of xanthohumol in combination with praziquantel.

Xanthohumol in combination with praziquantel could serve as potential anti-inflammation, anti-oxidant and antifibrotic agents in liver fluke-induced inflammation in relation to CCA development. Legend: $\downarrow$, regulate; $\perp$, inhibit. 
